\title{
RursuSpicae
}

Rusksuspicæ Transmission, réception et réécriture de textes, de l'Antiquité au Moyen Âge

\section{1 | 2018}

Parodies et pastiches antiques

\section{Avant propos à L'Humour d'Homère de Samuel Butler}

Preface of The Humour of Homer by Samuel Butler

\section{Didier Pralon}

\section{(2) OpenEdition \\ Journals}

\section{Édition électronique}

URL : http://journals.openedition.org/rursuspicae/354

DOI : $10.4000 /$ rursuspicae.354

ISSN : 2557-8839

\section{Éditeur :}

Université Nice-Sophia Antipolis, IRHT - Institut de recherche et d'histoire des textes

\section{Référence électronique}

Didier Pralon, « Avant propos à L'Humour d'Homère de Samuel Butler », RursuSpicae [En ligne], 1 | 2018, mis en ligne le 15 octobre 2018, consulté le 30 avril 2021. URL : http://journals.openedition.org/ rursuspicae/354; DOI : https://doi.org/10.4000/rursuspicae.354

Ce document a été généré automatiquement le 30 avril 2021.

RursuSpicae 


\section{Avant propos à L'Humour d'Homère de Samuel Butler}

Preface of The Humour of Homer by Samuel Butler

Didier Pralon

\section{NOTE DE L'ÉDITEUR}

Voir l'article de Samuel Butler : https://journals.openedition.org/rursuspicae/366

Merci à Dolorès Julia et à Dominique Merllié pour leurs relectures acribiques.

Dans l'écriture, l'humour, comme l'ironie, est difficile à déceler en l'absence de toute marque scripturaire; les guillemets ne lui suffisent pas. Il l'est aussi dans l'expression orale, s'il n'est pas appuyé par une mimique ou une formule complice. Il perd sa saveur s'il est explicité. Que penser alors des traits possibles d'humour dans les textes anciens, que ne caractérise pas l'étiquette "comiques »? L'usure du temps, le sérieux de la philologie conformiste et de l'enseignement classique en ont effacé la trace.

2 Samuel Butler (Langar-Nottinghamshire 1835-Londres 1902) associait l'humour à tous ses écrits, comme à ses comportements. Il fut un artiste éclectique et anticonformiste : " "écrivain philosophique" (selon le catalogue du Musée Britannique), et encore peintre, critique d'histoire religieuse, théoricien de la biologie générale, romancier, historien de l'art italien, humoriste, musicien, helléniste, savant shakespearien, poète, moraliste, mais surtout philosophe ou mieux encore, selon l'heureuse expression du critique [Jean Blum, alias Jean Florence, 1883-1915] qui, le premier, l'a présenté aux lettrés français, "humaniste" ", écrit Valery Larbaud, dans la préface à sa traduction française à Erewhon (Paris, Gallimard, 1920, p. 10). Au terme de ses études classiques à Cambridge, il refusa d'entrer dans les ordres, s'exila en Nouvelle Zélande le temps d'amasser, dans la pratique de l'élevage, un pécule qui lui permit, après son retour à Londres, de vivre décemment sans ostentation, publiant ses ouvrages de fiction, des études scientifiques et de critique littéraire et artistique à compte d'auteur. 
Il reprochait à ses congénères, comme à ses professeurs, d'être incapables de sentir la «chair» des textes sur lesquels portaient leurs études et leur enseignement, de proposer des traductions dépourvues d'âme: "Si vous voulez conserver l'esprit d'un ouvrage dont l'auteur est mort depuis longtemps, gardez-vous bien de le disséquer, de l'empailler et de le mettre sous verre. Plutôt mangez-le, digérez-le, et qu'il vive en vous, de votre vie à vous, quelle qu'elle soit, sans vous préoccuper du résultat. La différence entre la manière dont Andrew Lang traduit l'Odyssée et la manière dont je la traduis est la même qui existe entre embaumer un corps et engendrer un enfant. Il essaie de conserver un cadavre (car pour tous ceux qui ont besoin de la traduction de Lang, l'Odyssée est un cadavre), tandis que je tâche de créer une nouvelle forme vivante, et une forme vivante qui soit animée - autant que cela m'est possible -, par l'esprit de l'original, et non pas copiée sur sa forme... » (Carnets, traduction de Valery Larbaud, Gallimard, 1936, p. 203-204). Il condamne férocement les exégètes :

Les commentateurs d'Homère ont été si longtemps aveugles qu'ils ont voulu à toute

force qu'Homère l'ait été aussi. Ils ont étendu au poète leur propre cécité.

( "Homère et ses commentateurs", Carnets, p. 203).

Bien qu'il ne se soit jamais départi de son intérêt pour les poèmes homériques, il s'y plongea particulièrement à partir de 1892. Valery Larbaud, résumant la biographie de Butler composée par son ami H.F. Jones (Samuel Butler, Author of Erewhon (1835-1902,) a Memoir, by Henry Feston Jones, London, Macmillan, 1919), raconte l'aventure : «En même temps qu'il travaillait à la biographie de son grand-père, Butler poursuivait une autre étude : celle des poèmes homériques. En cherchant un sujet pour l'oratorio handélien ${ }^{1}$ qu'il voulait composer avec H.F. Jones, il avait songé aux aventures d'Ulysse, et, une fois ce sujet adopté, il avait voulu se rafraîchir la mémoire en relisant l'odyssée. On ne perd jamais le bénéfice d'une solide instruction classique: la lecture (à cinquante-quatre ans) de l'Odyssée le transporta. Dès lors il eut constamment Homère entre les mains et dans la pensée, au point de savoir les deux poèmes presqu'entièrement par cœur. Mais un esprit critique comme le sien ne devait pas se contenter de jouir de cette poésie : il y avait le "problème Homère" d'autant plus tentant qu'en s'y plongeant on ne pouvait que mieux goûter la poésie homérique. En 1892 il faisait au Collège des Travailleurs une conférence sur l'Humour d'Homère, et scandalisait tous les érudits habitués à ne voir dans les poèmes homériques qu'un "texte" capable seulement d'interprétations philologiques et non d'une interprétation humaine. Il avait déjà presque terminé une Traduction de l'odyssée lorsqu'il conçut une hypothèse touchant le lieu où une partie au moins du poème avait été composée : Trapani et le Mont Éryx, en Sicile, correspondaient à la description homérique du port de Schérie. Quelques mois plus tard il partait pour la Sicile, et ce fut dans une revue sicilienne, et en italien, qu'il donna la première ébauche de sa théorie de l'Odyssée (1893). Dès lors il fit de fréquents séjours à Trapani et dans la région, et son hypothèse se fortifia et grandit. Il parvint peu à peu, et en s'entourant de toutes les garanties scientifiques possibles, à établir que l'Odyssée avait dû être composée à Trapani. Et il ajouta à cette hypothèse ce corollaire un peu inattendu : que l'Odyssée était l'œuvre d'une femme, et cette femme... Nausikaa. Tel est le sujet de La Femme auteur de l'Odyssée (1897). Cependant, il avait approfondi aussi le problème de l'Iliade (dont il donna une traduction en 1898), mais sans lui apporter d'hypothèse nouvelle. En 1895 il avait fait un voyage en Grèce et dans la Troade pour se rendre compte par lui même de la valeur des conclusions auxquelles étaient arrivés les archéologues, et il reconnut que ces conclusions étaient acceptables. Sa traduction de l'Odyssée parut en 1900 » (Valery 
Larbaud, "Samuel Butler" in Erewhon, Paris, Gallimard, 1920, rééd. L'imaginaire, 1980, p. 24-25).

Pour être complet, il faut ajouter que Samuel Butler avait publié son "Iliade rendue en anglais" dès 1893, et avait préparé une traduction des Travaux et des jours d'Hésiode (publiée en 1924) ${ }^{2}$, qu'il ne publia pas lui-même, pas plus que son roman autobiographique: The way of all flesh (publié en 1904). Il composa aussi, avec son ami H.F. Jones, la cantate intitulée Ulysses, publiée elle aussi, après sa mort, en 1904.

Pour ce qui est de la conception qu'avait Samuel Butler de l'humour d'Homère, le dernier mot se lit dans les Carnets :

Quelque part en France, à Carcassonne, je crois, il y a un tombeau orné d'un basrelief qui représente les parents et les amis du défunt tout convulsés de douleur, les joues crevassées, et sanglotant comme les anges de Gaudenzio au Sacro-Monte de Varallo-Sesia. Mais dans un coin et invisible jusqu'à l'instant où le regard l'y va chercher, il y a un personnage qui crève de rire et se tient les côtes. Dans quelques endroits de l'Odyssée, et surtout dans les scènes entre Ulysse et Pénélope, j'imagine que ce rieur est là, invisible dans son coin («L'Odyssée et un tombeau de Carcassonne ", Carnets, p. 204).

\section{NOTES}

1. Butler orthographiait délibérément "Handel". Valery Larbaud respecte ce choix.

2. Le manuscrit est conservé à St John College, Cambridge.

\section{RÉSUMÉS}

Samuel Butler (1835-1902) reste aujourd'hui apprécié des amateurs de littérature victorienne pour plusieurs de ses nombreux ouvrages, notamment Erewhon (1872) et Ainsi va toute chair (publié de façon posthume en 1903 ; ces deux textes, comme d'autres, furent traduits en français par Valéry Larbaud). Il avait étudié les langues classiques à Cambridge et, durant toute sa vie, en conserva le goût, non sans anticonformisme. Le 30 janvier 1892, Il donna au Working Men's College de Londres, une conférence intitulée "The Humour of Homer". Le but en était de montrer que les philologues, gens trop uniment sérieux, n'avaient pas su percevoir la malice d'Homère et les tours qu'il joue à ses personnages. Il interprète de façon distanciée, citations et paraphrases à l'appui, dans l'Iliade, les relations des dieux entre eux, la querelle qui fait le sujet du récit, avec une prédilection pour Hector le Troyen (semblable à celle de Jean Giono, dans la préface méconnue qu'il écrivit en 1949 pour une réédition par Bordas de la traduction de L'Iliade qu'avait procurée, en 1715, Anne Dacier). Dans la composition de l'odyssée, il soupçonne la main d'une jeune femme, qui ne saurait être que Nausicaa supposée native de Trapani. Il prélude ainsi à son pamphlet littéraire The Authoress of the Odyssey, publié en 1897 (traduit en français par Christian 
Isidore Angelliaume : L'auteure de l'Odyssée, 2009, éd. L'Écluse Aval). Nous passerons sur les deux essais intermédiaires : "On the Trapanese origin of the Odyssey» [reprinted from The Eagle (dec. 1892) with a preface], Cambridge, Metcalfe, 1893 et « Was the Odyssey written by a woman?», in Collected Essays (vol. 2), New York, AMS press (The Shrewsbury edition of the works of Samuel Butler, vol. 19), 1968. On peut rappeler qu'il a aussi produit une traduction anglaise intégrale de l'Iliade (The Iliad of Homer Rendered into English Prose, 1898), et une autre de l'Odyssée (The Odyssey of Homer, 1900). Dans cette conférence, avant de développer sa perception de l'humour d'Homère dans les deux poèmes, $S$. Butler caractérise ainsi les thèmes propres de chacun, : "Les idéesforces de l'Iliade sont l'amour, la guerre et le pillage, quoique ce dernier sujet soit moins important que les deux autres. La note dominante de l'histoire c'est la séduction d'une femme et une querelle entre hommes pour sa possession [...] L'idée directrice de l'Odyssée est l'aveuglement de l'homme ».

Samuel Butler (1835-1902), who studied classical languages in Cambridge and offered a complete English translation of the Iliad (1898), and of the Odyssey (1900), remains popular among Victorian literary readers for his many works, including Erewhon (1872) and The Way of All Flesh (posthumously published in 1903), both of which were translated into French by Valery Larbaud. This text is the translation of a lecture given by Butler in 1892 (on January 30) at the Working Men's College in London. The aim is to show that philologists - being people excessively serioushad failed to perceive the malice of Homer and the tricks he plays to his characters. Butler interprets the interrelations between gods in the Iliad and the quarrel at the core of the story, with a predilection for Hector the Trojan (such as Jean Giono, who wrote in 1949 an inspired preface to a reedition of the translation of the Iliad procured in 1715 by Anne Dacier). He suspects the Odyssey to have been composed by a young woman, who could only be Nausicaa he supposed to be native of Trapani. This intuition was developed later in his literary pamphlet The Authoress of the Odyssey, published in 1897 (and translated into French by Christian Isidore Angelliaume : L'auteure de l'Odyssée, 2009, éd. L'Écluse Aval).

\title{
INDEX
}

Mots-clés : Butler (Samuel), Homère, Iliade, Odyssée

Keywords : Butler (Samuel), Homer, Iliad, Odyssey

\author{
AUTEUR \\ DIDIER PRALON \\ Philologue, est professeur émérite de langue et de littérature grecques à l'université d'Aix- \\ Marseille. Il est spécialiste de philosophie antique et de poésie ancienne. Son expertise s'étend de \\ la période archaïque à l'époque byzantine.
}

